\title{
A Unique Role for Fyn in CNS Myelination
}

\author{
Brian R. Sperber, ${ }^{1,2}$ Éilis A. Boyle-Walsh, ${ }^{1}$ Mark J. Engleka, ${ }^{1}$ Paul Gadue, ${ }^{1}$ Alan C. Peterson, ${ }^{4}$ Paul L. Stein, ${ }^{1}$ \\ Steven S. Scherer, ${ }^{3}$ and F. Arthur McMorris ${ }^{1,2}$
}

1The Wistar Institute, Philadelphia, Pennsylvania 19104, ${ }^{2}$ Graduate Group in Neuroscience and ${ }^{3}$ Department of Neurology, University of Pennsylvania, Philadelphia, Pennsylvania 19104, and ${ }^{4}$ Department of Neurology and Neurosurgery, Royal Victoria Hospital, McGill University, Montreal, Canada H3A 1 A1

We analyzed the role of Fyn tyrosine kinase in CNS myelination by using fyn ${ }^{-1-}$ null mutant mice, which express no Fyn protein. We found a severe myelin deficit in forebrain at all ages from $14 \mathrm{~d}$ to 1 year. The deficit was maximal at 1 month of age and was similar regardless of mouse strain background or whether it was determined by bulk isolation of myelin or by quantitation of myelin basic protein. To determine the cellular basis of the myelin deficit, we counted oligodendrocytes in tissue sections of mice expressing oligodendrocyte-targeted $\beta$-galactosidase, and we used light and electron microscopy to examine the number and morphology of myelinated fibers and size of myelinated CNS structures. All of these parameters were reduced in $\mathrm{fyn}^{-/-}$mice. Unexpectedly, there were regional differences in the myelin deficit; in contrast to forebrain, fyn ${ }^{-1-}$ cervical spinal cord exhibited no reduction in myelin content, number of oligodendrocytes, or number of myelinated fibers, nor was myelination delayed developmentally. We found that oligodendrocytes express Src, but there was no significant reduction of myelin content in null mutants lacking the Fynrelated kinases Src, Yes, or Lyn. Finally, we investigated the molecular features of Fyn that are required for myelination and found that a single amino acid substitution, which abolishes the tyrosine kinase activity of Fyn, resulted in a myelin deficit as great as that observed in the complete absence of Fyn protein. These results demonstrate that Fyn plays a unique role in myelination, one that requires its kinase activity.

Key words: myelin; oligodendrocyte; corpus callosum; spinal cord; tyrosine kinase; Fyn; Src; Lyn; Yes; development; knock-out
The regulation of myelination by oligodendrocytes in the CNS and Schwann cells in the PNS is poorly understood. We studied the possible role of Fyn, Src, Yes, and Lyn, four nonreceptor protein tyrosine kinases of the Src family, in myelination in the CNS.

Src family kinases have long been implicated in the regulation of cell growth and differentiation, and the family members Src, Fyn, Yes, Lyn, and Lck are expressed in the nervous system (Thomas and Brugge, 1997). Recent studies indicate that Fyn plays an important role in brain development and physiology; Fyn mutant mice exhibit disrupted hippocampal architecture, abnormal long-term potentiation, impaired spatial learning, and increased fearfulness and sensitivity to ethanol (Grant et al., 1992; Miyakawa et al., 1994, 1997). Fyn also plays a role in CNS myelination; mice homozygous for a Fyn- $\beta$-galactosidase $(\beta$-gal) fusion protein are hypomyelinated at 1 month of age (Umemori et al., 1994). Fyn is found in many brain areas, including glial cells in white matter tracts and in cultured oligodendrocytes, and its activity in brain is highest during the developmental period

\footnotetext{
Received Aug. 7, 2000; revised Dec. 20, 2000; accepted Jan. 5, 2001.

This work was supported by grants from The National Multiple Sclerosis Society and by National Institutes of Health Grant CA09171. S.S.S. was supported by National Institutes of Health Grant NS34528. P.L.S. is supported by National Institutes of Health Grants CA72806 and CA73796 and is a recipient of an Arthritis Foundation Investigator Award. We thank Dr. Ted Xu, Suzanne McGettigan, Neil Morton, and Trina Fernandes for their invaluable assistance.

Dr. Boyle-Walsh's present address: 1 Athlumney Castle, Navan, County Meath, Ireland.

Dr. Engleka's present address: Graduate Group in Cellular and Molecular Biology, University of Pennsylvania, Philadelphia, PA 19104.

Drs. Gadve's and Stein's present address: Department of Dermatology, University of Pennsylvania, Philadelphia, PA 19104.

Correspondence should be addressed to Dr. F. Arthur McMorris, The Wistar Institute, Philadelphia, PA 19104. E-mail: mcmorris@wistar.upenn.edu.

Copyright (C) 2001 Society for Neuroscience 0270-6474/01/212039-09\$15.00/0
}

approximately corresponding to the peak of myelination (Bare et al., 1993; Yagi et al., 1993; Umemori et al., 1994; Osterhout et al., 1999). Evidence suggests that the cell surface proteins myelinassociated glycoprotein (MAG), F3, and $120 \mathrm{kDa}$ neural cell adhesion molecule (NCAM120) may act as receptors coupled to Fyn (Umemori et al., 1994; Kramer et al., 1999).

Although Fyn has been shown to be important for CNS myelination, many important questions have not been addressed. Previous studies on Fyn mutants have been restricted to the first 2 months of age, raising the possibility that the hypomyelination only represents a temporary developmental delay. Also, previous studies have used Fyn mutants, which still express a fragment of the Fyn protein, raising the possibility that the fragment may bind to other molecules and disrupt their function rather than act as a true null. Furthermore, it is not known whether different CNS regions are equally affected in fyn mutant animals, nor is it known whether the myelin deficit results from a decrease in oligodendrocyte cell number or a reduction in the amount of myelin produced per oligodendrocyte. Finally, the possible role of other Src family kinase members in myelination has not been studied.

In the present study, we analyzed myelination in mice containing null mutations in each of four Src family kinases. In the $\mathrm{fyn}^{-/-}$mice, we investigated myelination at multiple time points, both by isolation and quantitation of myelin and by quantitation of the major CNS myelin protein, myelin basic protein (MBP). We used light and electron microscopy to analyze the number and morphology of myelin sheaths in several CNS regions, and we counted oligodendrocytes in several CNS regions to determine whether the hypomyelination in $f y n^{-1-}$ mice is the result of 
decreased oligodendrocyte numbers or a reduction in the amount of myelin produced by each oligodendrocyte. Finally, we investigated whether the tyrosine kinase activity of Fyn is essential for normal myelination.

\section{MATERIALS AND METHODS}

Mouse mutants and genotyping. Mice with mutations of fyn (Stein et al., 1992), src (Soriano et al., 1991), or yes (Stein et al., 1994) were bred, and homozygous offspring were used for experiments. $l y n^{-/-}$mice (Chan et al., 1997) were kindly provided by Dr. Ellen Puré (The Wistar Institute, Philadelphia, PA). Homozygous mice in which the wild-type fyn gene was replaced with a kinase-inactive mutant $f y n$ gene (FynK296R) by homologous recombination have been described elsewhere (P. L. Stein, unpublished data). Homozygous transgenic mice that express Escherichia coli $\beta$-galactosidase under the control of the mouse myelin basic protein promoter (referred to as $M b p$-lacZ mice; A. C. Peterson, unpublished data) were intercrossed with homozygous $f_{y n^{-1-}}$ mice to produce $f y n^{-1-}$ mice that also express the Mbp-lacZ transgene. Control mice for experiments involving these mutant mice were littermates, which were wild type at the fyn locus $\left(f y n^{+/+}\right)$and hemizygous or homozygous for lac $Z$ (expressing $\beta$-gal). The specificity of $\beta$-galactosidase expression exclusively within oligodendrocytes was verified by demonstrating the coexpression of $\beta$-galactosidase with multiple oligodendrocyte-specific markers in CNS tissue sections (A. C. Peterson, unpublished data). Wild-type $129 / \mathrm{Sv}, \mathrm{C} 57 \mathrm{BL} / 6 \mathrm{~J}$, or wild-type littermates of mutant mice were used as controls, as described in the individual experiments. Genotypes of $\mathrm{src}^{-1-}$ mice were determined according to methods described previously (Thomas et al., 1995). $\mathrm{fyn}^{-1-}$ mutant mice were genotyped using the following primers: Fyn reverse, 5'-GCAAAACAACCCACACAGAG3'; Fyn forward, 5'-AGCGAAACTGACAGAGGAGA-3'; and Neo2, TGGCTACCCGTGATATTGCT. The wild-type allele produces a 600 bp product, whereas a $450 \mathrm{bp}$ product is indicative of the mutant allele. Mice bearing the Mbp-lacZ transgene were identified by PCRbased genotyping using the following primers coding for a PCRamplification product of $\sim 600$ bp: Rob-1, 5'-GAAAACCCTGGCGTTACCCAACTT3'; and Rob-4, 5'-CTGAACTTCAGCCTCCAGTACAGC-3'. Mice were housed and all experimental procedures were conducted according to institutional, National Institutes of Health, and Institutional Animal Care and Use Committee guidelines and regulations.

Statistical analysis. Statistical analysis of data were performed using Student's $t$ test.

Determination of brain myelin content. Myelin from mouse forebrain was purified and quantitated by sucrose density gradient centrifugation, essentially as described by Norton and Poduslo (1973). In brief, mice were killed by $\mathrm{CO}_{2}$ inhalation, and the forebrain was quickly removed, weighed, and frozen in liquid nitrogen. The forebrain was defined by a coronal cut at the anterior border of the cerebellum. All brain structures rostral to that cut were included for determination of myelin content. For myelin isolation, the frozen brains were homogenized in $0.32 \mathrm{M}$ sucrose and centrifuged on a 0.32:0.85 $\mathrm{m}$ discontinuous sucrose gradient at $82,500 \times g$ for $30 \mathrm{~min}$. Myelin was collected from the interface, osmotically shocked in deionized water to release contaminants from within myelin vesicles, and recentrifuged over a second discontinuous sucrose gradient as above. The myelin was again collected from the interface and repeatedly washed with deionized water and pelleted to remove residual sucrose. The myelin was frozen at $-80^{\circ} \mathrm{C}$, lyophilized in tared tubes, and weighed.

Western blot analysis. Mice were killed by $\mathrm{CO}_{2}$ inhalation, and the forebrain was removed and homogenized in lysis buffer $(1 \%$ Nonidet P-40, $0.5 \%$ sodium deoxycholate, $0.1 \%$ SDS, $100 \mu \mathrm{g} / \mathrm{ml}$ PMSF, $57 \mu \mathrm{g} / \mathrm{ml}$ aprotinin, $1 \mathrm{~mm}$ sodium orthovanadate, and $100 \mu \mathrm{M}$ leupeptin, in PBS). Homogenate was then incubated on ice for $30 \mathrm{~min}$ and centrifuged at $10,000 \mathrm{rpm}$ for $10 \mathrm{~min}$ at $4^{\circ} \mathrm{C}$. Equal amounts of lysate ( $40 \mu \mathrm{g}$ of protein) were separated by electrophoresis on $12 \%$ SDS-PAGE and transferred to polyvinylidene difluoride membranes. After blocking overnight in block-

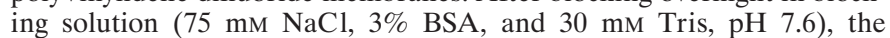
membranes were probed with antibodies against MBP (McMorris et al., 1981), neurofilament light chain (NF-L) (Dr. Virginia Lee, University of Pennsylvania), and glyceraldehyde 3-phosphate dehydrogenase (GAPDH) (Chemicon, Temecula, CA), followed by treatment with ${ }^{125}$ Ilabeled protein A (NEN Life Science Products, Boston, MA). The amount of MBP, NF-L, and GAPDH was quantified using a Molecular Dynamics (Eugene, OR) PhosphorImager.

For Western blot analysis of Src, immunopurified oligodendroglial cells (see below) were washed with cold PBS, and protein extracts were prepared in the lysis buffer described above. Equal amounts of lysate (70 $\mu \mathrm{g}$ of protein) were separated on $8 \%$ SDS-PAGE and transferred as above. After blocking overnight, the filters were probed with a monoclonal antibody against Src (1:500) (Oncogene Sciences, Uniondale, NY) and then incubated with $15 \mu \mathrm{g} / \mathrm{ml}$ rabbit anti-mouse IgG (Rockland Immunochemicals, Gilbertsville, PA). Then membranes were incubated with ${ }^{125}$ I-labeled protein A, and bands were visualized using a Molecular Dynamics PhosphorImager.

$\beta$-Galactosidase staining. Mice were deeply anesthetized and transcardially perfused with $0.9 \% \mathrm{NaCl}$, followed by fixative $(0.5 \%$ paraformaldehyde and $2.5 \%$ glutaraldehyde in $0.1 \mathrm{M}$ sodium phosphate buffer, $\mathrm{pH}$ 7.5). Brains and cervical spinal cords were then removed, further dissected to isolate only the desired tissue, and incubated in fixative for $1 \mathrm{hr}$ at $4^{\circ} \mathrm{C}$. Tissue blocks were then immersed in $30 \%$ sucrose in $0.1 \mathrm{M}$ sodium phosphate buffer, $\mathrm{pH} 7.5$, for $48 \mathrm{hr}$ at $4^{\circ} \mathrm{C}$, embedded in OCT freezing medium (Tissue Tek, Miles Inc., Elkhart, IN), and frozen in an acetonedry ice slurry. Cryosections (6- $\mu$ m-thick) were cut and allowed to adhere to glass slides (SuperFrost Plus; Fisher Scientific, Pittsburgh, PA). Sections then were dried for $15 \mathrm{~min}$ at $37^{\circ} \mathrm{C}$ and incubated in 5-bromo-4chloro-3-indolyl- $\beta$-D-galactopyranoside (X-gal) staining solution $[0.4$ $\mathrm{mg} / \mathrm{ml} \mathrm{X-gal} \mathrm{(Boehringer} \mathrm{Mannheim,} \mathrm{Indianapolis,} \mathrm{IN),} 3 \mathrm{~mm}$ potassium ferricyanide, $3 \mathrm{~mm}$ potassium ferrocyanide, and $2 \mathrm{mM} \mathrm{MgCl}_{2}$, in $0.1 \mathrm{M}$ sodium phosphate buffer, $\mathrm{pH} 7.5$ ] for $30 \mathrm{~min}$ at $37^{\circ} \mathrm{C}$, after which they were washed with PBS, mounted under coverslips in aqueous mounting medium, and observed by conventional light microscopy. The crosssectional areas of spinal cord and corpus callosum were determined by capturing a light microscope image of the structure with a digital camera and determining its area in pixels, using Adobe Photoshop (Adobe Systems, San Jose, CA). A microruler was observed and measured by the same procedure to convert area in pixels to square millimeters.

Electron microscopy. Postnatal day 0 (P0), P14, and P28 mice were deeply anesthetized and transcardially perfused with $0.9 \% \mathrm{NaCl}$, followed by $3 \%$ glutaraldehyde in $0.1 \mathrm{~m}$ sodium phosphate buffer, $\mathrm{pH}$ 7.5. Brain, optic nerve, and cervical spinal cord were then removed, trimmed to minimize excess tissue, and incubated overnight in $3 \%$ glutaraldehyde in $0.1 \mathrm{M}$ sodium phosphate buffer, $\mathrm{pH}$ 7.5. Tissue blocks were then washed in $0.1 \mathrm{M}$ sodium phosphate buffer and incubated $1.5 \mathrm{hr}$ in $2 \%$ osmium tetroxide. Blocks were washed in $0.1 \mathrm{M}$ sodium phosphate buffer, $\mathrm{pH} 7.5$, dehydrated in ethanol, and equilibrated in Epon $(44.2 \%$ Embed $812,35.4 \%$ dodecenylsuccinic anhydride, $17.7 \%$ nadic methyl anhydride, and $2.7 \%$ benzyldimethylamine). Sections $(0.5-\mu \mathrm{m}$-thick) for light microscopy were cut using an ultramicrotome and stained with toluidine blue. Thin sections for electron microscopy were cut using an ultramicrotome, stained with $4 \mathrm{mg} / \mathrm{ml}$ uranyl acetate in $95 \%$ ethanol and 0.4 $\mathrm{mg} / \mathrm{ml}$ lead citrate, and analyzed using a Zeiss (Oberkochen, Germany) electron microscope. For quantitative analysis of the number of myelinated fibers in the corpus callosum, we counted myelinated fibers and the total number of axons in 10 closely spaced, nonoverlapping fields of 1680 and $340 \mu \mathrm{m}^{2}$, respectively, from sagittal sections in the genu of the corpus callosum.

In vitro preparation of purified mouse oligodendroglial cells. Postnatal day 1 C57BL/6J or $f_{y n}{ }^{-1-}$ mouse pups were decapitated, and their forebrains were removed and minced in wash medium (25 mM HEPES,

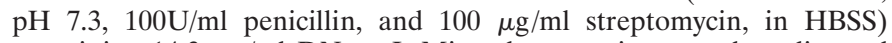
containing $14.3 \mu \mathrm{g} / \mathrm{ml}$ DNase I. Minced suspension was then digested with $1 \%$ trypsin in calcium-free PBS for $70 \mathrm{~min}$ in a $37^{\circ} \mathrm{C}$ incubator with shaking at $250 \mathrm{rpm}$. The suspension was then centrifuged at $500 \mathrm{rpm}$ $(75 \times g)$ for $10 \mathrm{~min}$, resuspended in wash medium containing $14.3 \mu \mathrm{g} / \mathrm{ml}$ DNase I, and centrifuged again for $10 \mathrm{~min}$. Cells were then resuspended in OM-5 culture medium (Raible and McMorris, 1990), which contains $10 \%$ serum, sequentially passed through two $70 \mu \mathrm{m}$ pore-sized cell strainers (Falcon Labware, Lincoln Park, NJ), plated in $75 \mathrm{~cm}^{2}$ tissue culture flasks at $30 \times 10^{6}$ cells per flask, and incubated in $10 \% \mathrm{CO}_{2}-90 \%$ air at $37^{\circ} \mathrm{C}$.

Oligodendrocytes and their precursors were purified by immunopanning using monoclonal antibodies against cell surface antigens, essentially as described previously (Barres et al., 1992; Vemuri and McMorris, 1996). Ran-2, an antibody that recognizes astrocytes (Eisenbarth et al., 1979; Sommer and Schachner, 1981), was prepared as a tissue culture supernatant from hybridoma cells. Briefly, an enriched population of oligodendrocytes and precursor cells was isolated by differential shake off of 7-d-old mouse glial cell cultures essentially as described by McCarthy and de Vellis (1980). The cell suspension was then incubated for $30 \mathrm{~min}$ intervals over a series of three bacteriological Petri dishes (Fisher Sci- 
entific) coated with Ran-2 monoclonal antibody. During this time, astrocytes (which express the Ran-2 surface antigen) and microglia [which are very adhesive and bind to immunoglobulins via their $\mathrm{F}(\mathrm{c})$ receptors] attached to the Ran-2 antibody on the surface, whereas oligodendroglia remained in suspension. A sample of nonadherent cells was incubated for 30 min with $1 \mu \mathrm{M}$ prostaglandin $\mathrm{E}_{1}\left(\mathrm{PGE}_{1}\right)$ and $1 \mu \mathrm{M} \mathrm{PGE}_{2}$ and immunostained with antibody against CAMP to determine whether astrocytes or microglia were still present. Previous work from our laboratory has shown that oligodendroglia do not elevate cAMP in response to this treatment, whereas non-oligodendroglial cells in the cultures (astrocytes and microglia) respond robustly and are intensely immunostained (A. P. Wiemelt and F. A. McMorris, unpublished observations). No cells ( $<1$ of 2000) were positive for cAMP immunofluorescence. The remaining nonadherent cells were centrifuged at $500 \mathrm{rpm}(75 \times \mathrm{g})$ for $10 \mathrm{~min}$ and prepared for Western blot analysis as described above.

\section{RESULTS}

\section{Mice that lack Fyn are irreversibly myelin-deficient}

As described in detail previously (Stein et al., 1992), fyn ${ }^{-1-}$ mutant mice were derived by disrupting the normal fyn gene using a targeting construct that deleted exon 2, the first coding exon, which contains the initiator methionine. The resulting $f y n^{-1-}$ mice express no detectable Fyn protein (Stein et al., 1992). We analyzed myelin content of forebrain in $\mathrm{fyn}^{-/-}$and agematched C57BL/6J (B6) control mice at time points ranging from P14 to P385 and found a myelin deficit at all time points analyzed (Fig. 1A). The deficit was most pronounced at postnatal day 26 $(52 \%)$ and persisted even at ages beyond 1 year. From P55 to $\mathrm{P} 385$, the myelin deficit remained relatively constant and was significantly different from controls at all time points, with the exception of $\mathrm{P} 283$ (Fig. $1 A$ ).

The fyn $^{-1-}$ mutant mice we used were derived from $129 \mathrm{~Sv}$ embryonic stem cell chimeras that were crossed to B6 mice to produce hybrid animals, which were then maintained by interbreeding. Thus, the genotype of the mutant mice contained contributions from the B6 and 129/Sv (129) strains but was not inbred or even uniform between littermates, making it problematic to select an appropriate strain for the control animals. For most experiments, we used age-matched inbred B6 mice as controls. Because the myelin content data demonstrated a clear difference between the inbred B6 controls and the non-inbred $f_{y n}{ }^{-1-}$ B6,129 mice, and because the SEs at a given age were of a similar magnitude within the control and $f y n^{-1-}$ groups, we thought it unlikely that the observed myelin deficit was primarily attributable to strain differences. To address this issue further and determine whether the myelin deficit is indeed the result of differences at the fyn locus, we also quantitated myelin content of $\mathrm{fyn}^{-1-}$ and $f y n^{+/+}$mice that were maintained on the inbred 129Sv background (Stein et al., 1992). These $\mathrm{fyn}^{-/-} 129$ mice were analyzed for myelin content at P26 and compared with inbred 129 control mice of the same age. As shown in Figure $1 E$, the $\mathrm{fyn}^{-1-} 129$ mice had a $37 \%$ deficit in forebrain myelin content at P26 $(p<0.005)$, in agreement with the 52\% deficit observed in the $f_{y n}{ }^{-1-}$ B6,129 mice at the same age (Fig. $1 E$ ). Thus, the difference in myelin content observed in $f_{y n}{ }^{-1-}$ mice compared with controls is attributable to differences at the fyn locus and not to differences in genetic background.

Myelin content was also assessed in whole homogenates by quantitation of the amount of MBP, a myelin-specific protein that accounts for $30-35 \%$ of the total protein of CNS myelin (Lees and Brown, 1984). MBP was assayed on Western blots using P28 forebrain or spinal cord homogenate from $\mathrm{fyn}^{-/-}$and B6 control mice. Blots were probed with a combination of antibodies against $\mathrm{MBP}$, the neuron-specific protein NF-L and the ubiquitously expressed GAPDH. Brain GAPDH content per milligram of protein was unchanged in $f y n^{-1-}$ mouse brain and spinal cord (both, $p>0.1$ ) when compared with B6 controls (data not shown). When the amount of MBP was normalized to GAPDH, MBP was found to be significantly reduced in $f y n^{-1-}$ brain compared with B6 controls $(p<0.01)$ (Fig. 1B,C), and the magnitude of the deficit (40\%) was comparable with that determined by myelin isolation $(52 \%)$. A similar deficit was observed when the amount of MBP was normalized to protein loading (data not shown). Therefore, the myelin deficit determined by quantitation of purified myelin cannot be attributed to errors encountered during myelin isolation and purification. Unexpectedly, the amount of NF-L per milligram of protein was reduced by $18.3 \%$ in brains of $f y n^{-1-}$ mice compared with B6 controls $(p<0.01)$ (Fig. 1B,C).

Both methods of quantitation of myelin content revealed a deficit of myelin content in forebrain. In contrast, MBP content in P28 $\mathrm{fyn}^{-1-}$ mice was not significantly reduced in spinal cord, regardless of whether the data were normalized to GAPDH $(p>$ 0.5 ) (Fig. $1 B, C)$ or to total protein $(p>0.1$ ) (data not shown).

\section{Fyn is unique among Src-family kinases in being essential for myelination}

Fyn is one of nine known members of the Src family of protein tyrosine kinases, which are highly similar in amino acid sequence, except in the N-terminal "unique" domain (Brown and Cooper, 1996). In addition to Fyn, four other Src family members, Src, Yes, Lyn, and Lck, have been shown to be expressed in brain (Thomas and Brugge, 1997); Fyn and Lyn are expressed in oligodendrocytes (Kramer et al., 1999; Osterhout et al., 1999). Therefore, we sought to determine whether Src, Yes, or Lyn are essential for normal myelination. Here we demonstrate, by Western blot analysis of lysates of highly purified cultures of B6 mouse oligodendrocytes, that $\mathrm{Src}$ is also expressed in oligodendrocytes (Fig. 1D). In these cultures, no astrocytes or microglia could be detected (less than 1 of 2000 cells scored) by the highly sensitive method of cAMP immunofluorescence of $\mathrm{PGE}_{1} / \mathrm{PGE}_{2}$ stimulated cells (see Materials and Methods). The specificity of the Src antibody was demonstrated by the lack of a signal in src $^{-1-}$ brain samples (Fig. 1D).

Analysis of the myelin content of $s \mathrm{c}^{-1-}, y e \mathrm{~s}^{-1-}$, and $l y n^{-1-}$ mice at P28 revealed that there is no significant myelin deficit in these mutants (Fig. 1E). Therefore, among Src family kinases, Fyn plays a unique role in myelination.

\section{Morphological analysis of myelin in fyn ${ }^{-1-}$ CNS}

To determine whether myelin structure, as well as myelin content, is compromised in $\mathrm{fyn}^{-/-}$animals, we analyzed P28 $\mathrm{fyn}^{-1-}$ mouse myelin profiles in three separate CNS white matter areas: corpus callosum, optic nerve, and spinal cord. To ensure that we were analyzing similar regions in each animal, we focussed our analysis on discrete locations within each structure; in the brain, we analyzed sagittal sections of the genu of the corpus callosum, and in the spinal cord, cross-sections at the level of the cervical enlargement. The optic nerve is sufficiently small and homogeneous so that we analyzed entire cross-sections of its intracranial segment.

In sagittal sections of corpus callosum from P28 fyn ${ }^{-1-}$ mice, we observed a widespread reduction in the number of myelin sheaths (Fig. 2). In 10 closely spaced, nonoverlapping fields from the genu, we observed a $77 \%$ reduction in the number of myelin profiles $(p<0.0001)$ (Fig. $2 E)$. This reduction was not the result of an overall decrease in the number of axons, because the 
Figure 1. Myelin content is reduced in forebrain of $f y n^{-1-}$ mice but not in $s r c^{-1-}$, yes ${ }^{-1-}$, or $l y n^{-1-}$ mice. $A$, Myelin content is reduced in $f y n^{-1-}$ mouse forebrain. Myelin from C57BL/6J,129Sv $\mathrm{fyn}^{-1-}$ or $\mathrm{C} 57 \mathrm{BL} / 6 \mathrm{~J} \mathrm{fyn}^{+/+}$control mouse forebrain was isolated by sucrose density centrifugation and quantified at P14, P26, P55, P95, P283, or P385. Each point represents at least six mice. Data are expressed as milligrams of myelin (dry weight) per gram of brain (wet weight). $f y n^{-1-}$ values are significantly different from control at days P26, P55, $\mathrm{P} 95$, and $\mathrm{P} 385(p<0.05)$. $B$, Western blot analysis of MBP content in $f y n^{-1-}$ forebrain and spinal cord. Samples of lysate containing $30 \mu \mathrm{g}$ of protein from P28 $f y n n^{-1-}$ and control mouse forebrain or spinal cord were separated on a $12 \%$ polyacrylamide gel and transferred to a PVDF membrane, and the blot was probed with antibodies against NF-L, GAPDH, and MBP, followed by ${ }^{125} \mathrm{I}-$ protein $\mathrm{A}$. Bands were visualized by autoradiography. $C$, The amount of radioactivity in individual bands of the blot shown in $B$ was quantified by PhosphorImager analysis, and the amount of MBP or NF-L was normalized to the amount of GAPDH in the same lane. Each bar represents the mean \pm SEM normalized MBP or NF-L from four different animals, expressed as percent of that in $\mathrm{fyn}^{+/+}$controls. The amount of MBP and NF-L is significantly different from controls in forebrain of $\mathrm{fyn}^{-1-}$ mice $(p<$ 0.01 and $p<0.05$, respectively) but not in spinal cord $(p>0.5) . D$, Src is expressed in oligodendrocytes. Samples of lysate containing $80 \mu \mathrm{g}$ of protein from B6 mouse forebrain (WT brain), $s \mathrm{sc}^{-1-}$ mouse forebrain, highly purified B6 oligodendrocytes (WT oligos), and highly purified $f y n^{-1-}$ oligodendrocytes were separated on a $12 \%$ polyacrylamide gel and transferred to a PVDF membrane, and the blot was probed with monoclonal antibody against Src. Bands were visualized by autoradiography. Src immunoreactivity is evident in all samples except lysate from $\mathrm{src}^{-1-}$ mouse forebrain. $E$, Myelin is reduced in forebrain of $f y n^{-1-}$ mice but not in forebrain of $\mathrm{src}^{-1-}$, yes ${ }^{-1-}$, or $l y n n^{-1-}$ mice. Myelin was isolated from forebrain of $f y n^{-1-}, \mathrm{src}^{-1-}, \mathrm{yes}^{-/-}, \mathrm{lyn}^{-1-}$, and control mice by sucrose density gradient centrif ugation and quantified. Data, calculated as milligrams of myelin (dry weight) per gram of brain (wet weight), are shown as percent of control. Each bar represents the mean of at least five mice; error bars represent SEM. The myelin content of B6,129 $\mathrm{fyn}^{-/-}$mouse forebrain at P26 is shown relative to age-matched B6 control mice. In addition, myelin content of strain 129Sv fyn $n^{-1-}$ mouse forebrain at P26 is shown compared with age-matched 129Sv control mice. In both genetic backgrounds, myelin content was significantly less in $\mathrm{fyn}^{-/-}$mice than in controls $(p<0.05)$. Myelin content of $\mathrm{src}^{-/-}$, yes $^{-/-}$, and lyn ${ }^{-/-}$mice at day P28 is shown compared with that of age-matched control mice. There is no significant difference in myelin content when comparing mice lacking Src, Yes, or Lyn and control mice $(p>0.05)$.

percent of axons that were myelinated also was markedly reduced, by $89 \%$. Although the number of myelin sheaths was reduced, they had a normal ultrastructure (Fig. 2C). Moreover, the size distribution of axons appeared to be normal, and myelin sheath thickness appeared appropriate for axonal caliber (Fig. 2).

Analysis of optic nerve from $f_{y n}{ }^{-1-}$ mice revealed a $15 \%$ reduction in cross-sectional area relative to controls $(p<0.005)$, consistent with a reduction in myelin content, although no striking reduction in abundance of myelin profiles comparable with that in corpus callosum was apparent (Fig. 3). However, in morphology, fyn ${ }^{-1-}$ mouse myelin profiles were indistinguishable from control (Fig. 3).
To determine whether there were structural abnormalities in myelin from the spinal cord of $f y n^{-/-}$mice, we analyzed white matter tracts at the level of the cervical enlargement. At P28, we observed no decrease in the abundance of myelin sheaths, and no morphological deficits (Fig. 4E,F). The dorsal, ventral, and lateral funiculi all appeared normal. Similar results were observed at P14 (Fig. 4C,D). Although much less myelin was present in both controls and knock-outs at this earlier time point, no morphological abnormalities could be detected. To determine whether there is a delay in the onset of myelination in the absence of Fyn, $f_{y} n^{-1-}$ and control, mouse spinal cords were analyzed at $\mathrm{P} 0$, the age at which myelin profiles can first 



Figure 2. The corpus callosum is hypomyelinated in $\mathrm{fyn}^{-1-}$ mice. Electron micrographs of the genu of the corpus callosum, in midline sagittal sections of control $(B, D)$ and $f y n^{-1-}$ $(A, C)$ mice at $28 \mathrm{~d}$ of age. The reduction in number of myelin sheaths in the $\mathrm{fyn}^{-1-}$ mice is evident (compare $A$ with $B$, and $C$ with $D$; arrows indicate representative myelin sheaths). Numerous unmyelinated axons (arrowheads) are evident in both $f y^{-/-}$and $f y n^{+/+}$mouse corpus callosum $(C, D)$, but they account for a greater proportion of total axons in the fyn ${ }^{-1-}$ mice. Scale bars: $A, B, 10 \mu \mathrm{m} ; C, D, 2 \mu \mathrm{m}$. $E$, Myelinated fibers and the total number of axons were counted in 10 randomly selected, nonoverlapping fields of $1680 \mu^{2}$ (myelinated fibers) and $340 \mu \mathrm{m}^{2}$ (axons) from midline sagittal sections in the genu of the corpus callosum. The number of myelinated axons per square millimeter and the percent of total axons that are myelinated were calculated and are shown in the figure as percent of control. Values represent the mean \pm SEM of data from three different animals of each genotype. Both the number of myelin profiles and the percent of axons that are myelinated are significantly reduced in the $f y n^{-1-}$ mouse corpus callosum when compared with controls $(p<$ $0.0001)$.

Figure 3. Myelination in the optic nerve of fyn $^{-1-}$ and control mice. Light micrographs of the intracranial portion of the optic nerve, in $0.5-\mu \mathrm{m}$-thick toluidine blue-stained crosssections of $f y n^{-1-}(A, C)$ and control $(B, D)$ mice at $28 \mathrm{~d}$ of age. The significant reduction in cross-sectional area in fyn ${ }^{-1-}$ mice $(p<0.005)$ is evident at low-power magnification $(A, B)$. The morphology of myelin from $\mathrm{fyn}^{-1-}$ mice appears indistinguishable from control at higher magnification $(C, D)$. Each micrograph is representative of sections from three different animals. Scale bars: $A, B, 50 \mu \mathrm{m} ; C, D, 10 \mu \mathrm{m}$. be detected in control mouse spinal cord. Myelin profiles were present in $f y n^{-1-}$ spinal cord at P0 (Fig. 4A,B) and appeared indistinguishable from control in both their abundance and morphology. Therefore, the development, onset, and maturation of myelin sheaths in the cervical spinal cord was unaffected in $\mathrm{fyn}^{-/-}$mice. 

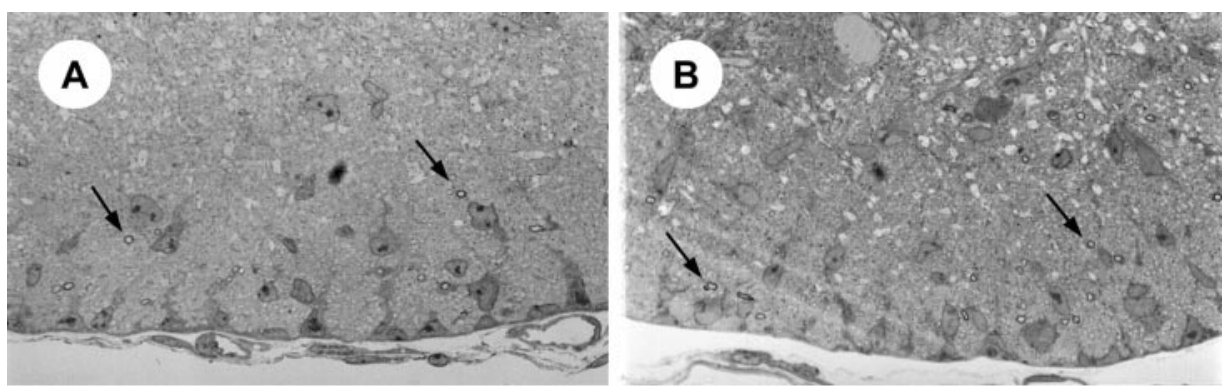

Figure 4. Myelination in spinal cord of $f y n^{-/}$ and control mice. Light micrographs of the ventral funiculus in the cervical enlargement of the spinal cord, in $0.5-\mu \mathrm{m}$-thick toluidine bluestained cross-sections from $f_{y n}{ }^{-1-}(A, C, E)$ and control $(B, D, F)$ mice. Myelin profiles are evident in both $f y^{-1-}$ and control mouse ventral funiculus at P0 (arrows in $A$ and $B$ ). Similarly, sections of ventral funiculus from $\mathrm{P} 14(C$, $D)$ and $\mathrm{P} 28(E, F)$ mice demonstrate that there is no appreciable difference in myelin sheath abundance or morphology in $f y n^{-/-}$mice $(C$, $E)$ compared with controls $(D, F)$. Each micrograph is representative of sections from three different animals. Scale bar, $20 \mu \mathrm{m}$.
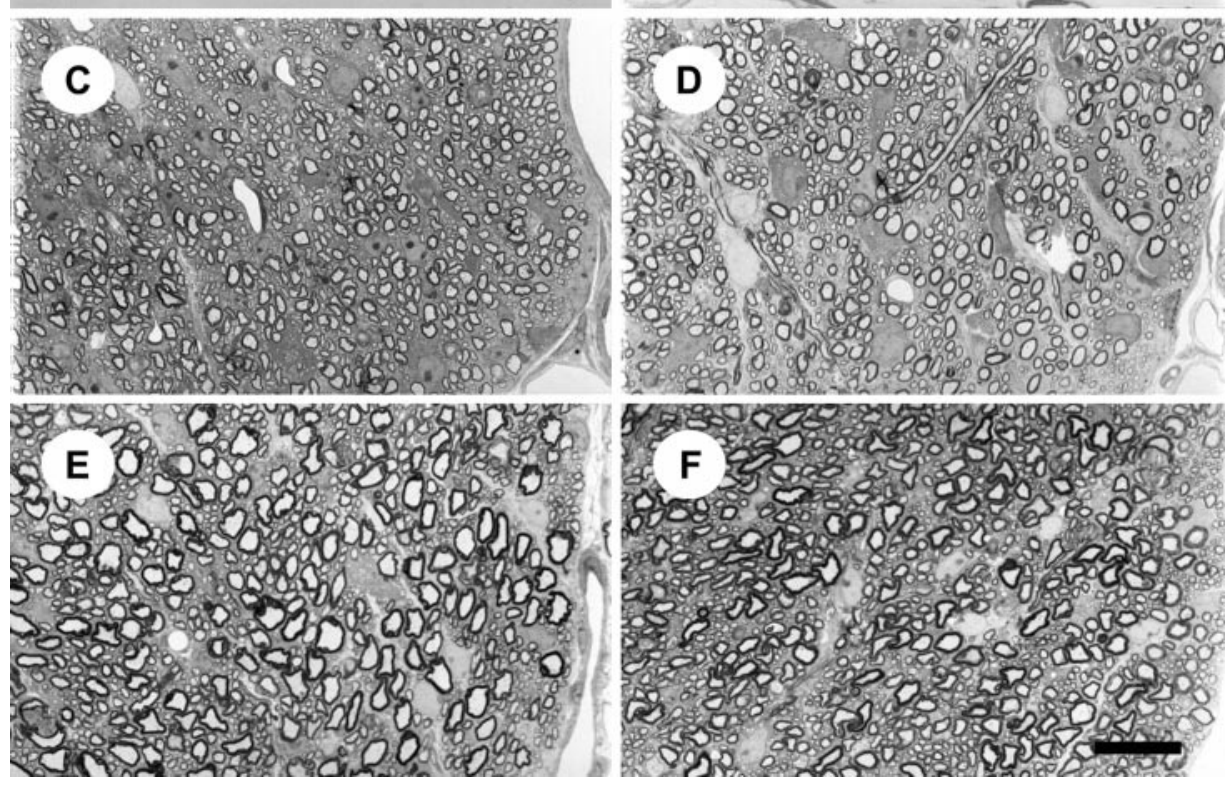

\section{Oligodendrocyte numbers are reduced in $\mathrm{fyn}^{-1-}$ corpus callosum but not in spinal cord}

The deficit in myelin content of $f y^{-1-}$ mice might be the result of a reduction in the number of oligodendrocytes, a reduction in the amount of myelin elaborated by an individual oligodendrocyte, or both. To investigate this issue, we analyzed oligodendrocyte number in selected CNS areas of $f y^{-1-}$ and control mice. To facilitate identification and accurate counting of oligodendrocytes, we used transgenic mice in which expression of bacterial lac $Z$ gene (which encodes $\beta$-gal) was targeted specifically to oligodendrocytes with a mouse MBP promoter transgene. A nuclear localization signal was placed in-frame with $\beta$-gal, and as shown in Figure 5, $\beta$-gal activity was clearly evident in the nuclei of cells expressing the transgene and was also detected in the soma and to a lesser extent in cellular processes and myelin. $M b p-l a c Z$ transgenic mice were intercrossed with $f y n^{-1-}$ mice to produce mice that express $\beta$-gal in their oligodendrocytes and are null $(-/-)$ at the fyn locus. Cryosections of forebrain and spinal cord were stained with X-gal, and the total number of oligodendrocytes was counted in each section.

In coronal sections of forebrain, we observed a significant $29 \%$ reduction in the average number of oligodendrocytes per section within a fixed width $(0.8 \mathrm{~mm}$ at the midline $)$ of the corpus callosum $(p<0.005)$ (Fig. $5 E)$. In addition, the thickness of the corpus callosum was reduced in all $f y n^{-1-}$ mice analyzed (Fig. $5 A, C)$, but the number of mice analyzed was insufficient for statistical testing of this parameter. In contrast, in cervical spinal cord, the number of oligodendrocytes was not detectably different between $f_{y n}{ }^{-1-}$ and littermate control mice (Fig. 5E). When expressed either as the number of oligodendrocytes per cross- section of spinal cord or the number of oligodendrocytes per square millimeter, there was no significant difference between $f_{y n}{ }^{-1-}$ mice and littermate controls $(p>0.2$ and $p>0.4$, respectively) (Fig. 5E).

\section{The myelin deficit in fyn ${ }^{-/}$mice results from the absence of the kinase activity of Fyn}

Because of the important role of protein phosphorylation in signaling and cellular regulation, studies on the function of Fyn and other protein tyrosine kinases usually focus on their kinase activity. However, there is increasing evidence that many kinases, including Src family kinases, also may have other important functions in cells [e.g., as adapter molecules, via their Src homology 2 (SH2), SH3, or "unique" domains, or by competing with other molecules for binding to putative partners]. To determine whether the tyrosine kinase activity of Fyn is essential for normal myelination, we analyzed myelin content in mice expressing fulllength Fyn containing a point mutation at amino acid 296 (lysine to arginine), which prevents ATP binding, thus abolishing kinase activity. Brains from these Fyn K296R mice were analyzed for myelin content. Relative to B6 control, myelin content in Fyn K296R mouse brain was reduced by 66\% $(p<0.0005)$ (Fig. 6), which is similar to the deficit observed in the complete absence of Fyn protein (Fig. 1). Therefore, the kinase activity of Fyn is necessary for normal myelination.

\section{DISCUSSION}

In the present study, we analyzed myelination in the complete absence of Fyn, using $f y n^{-1-}$ mice that make no detectable Fyn 

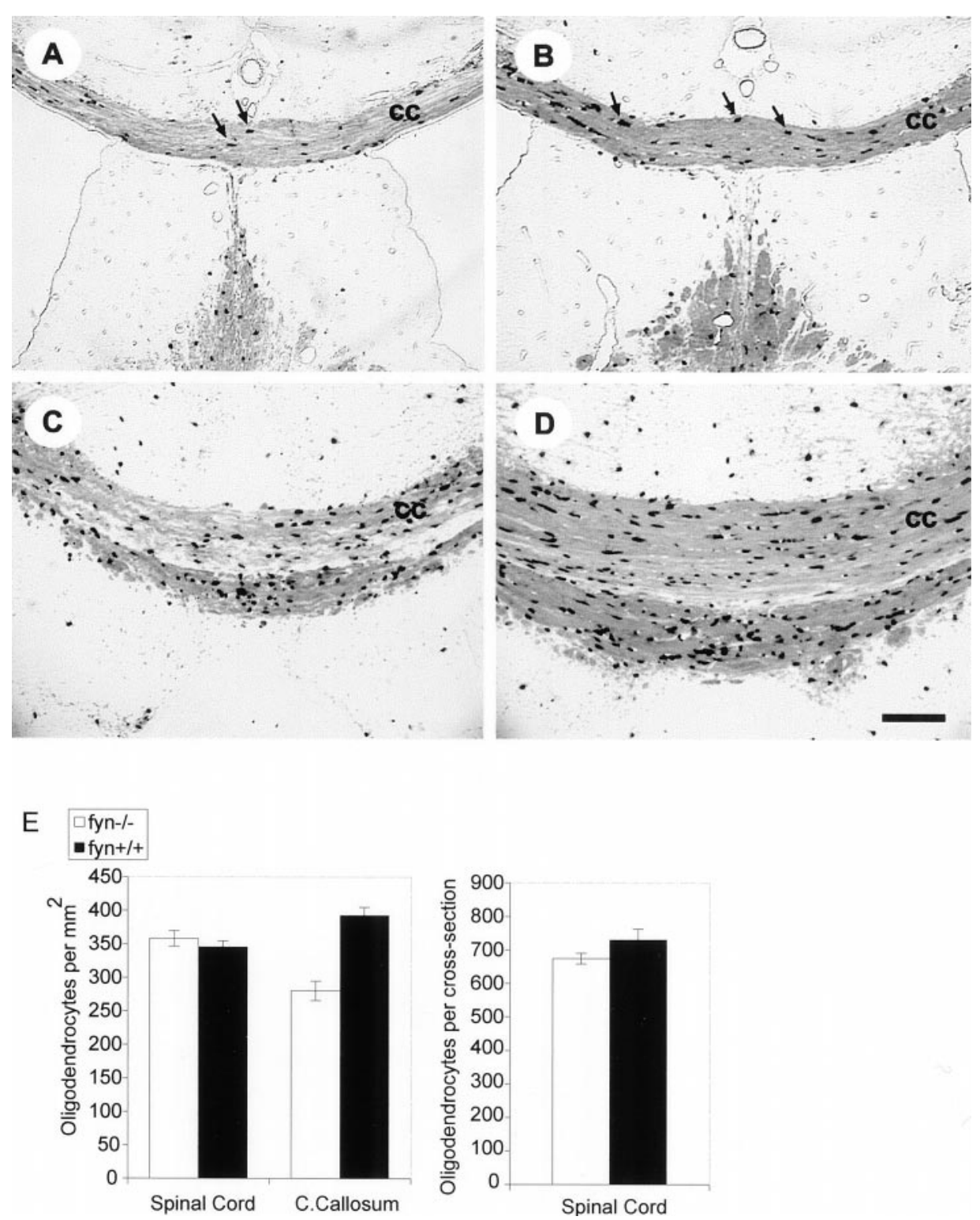

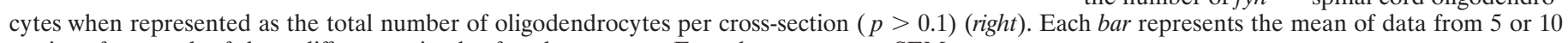
sections from each of three different animals of each genotype. Error bars represent SEM.

Figure 5. Oligodendrocyte number and myelination are reduced in the corpus callosum of fyn $^{-1-}$ mice. $A-D$, Light micrographs of $10-$ $\mu \mathrm{m}$-thick coronal cryosections from $\mathrm{yyn}^{-1-}(A$, $C)$ and control $(B, D)$ mice. Control and $f y n^{-1-}$ mice carry an $M b p$-lac $Z$ transgene that targets $\beta$-galactosidase expression to oligodendrocytes, resulting in dark staining of oligodendrocyte nuclei and cell bodies and lighter staining of myelin in the X-gal-stained sections. Darkly stained oligodendrocytes are concentrated in the corpus callosum and surrounding white matter regions (arrows in $A$ and $B$ ). Fewer oligodendrocytes are apparent in $f y n^{-1-}$ corpus callosum $(c c)$ at the level of the ventral hippocampal commissure (compare $A$ with $B$ ). Similarly, reduced numbers of oligodendrocytes are evident at the level of the habenula, in which a reduction in corpus callosum thickness is also apparent (compare $C$ with $D$ ). Micrographs are representative of sections from three different animals. Scale bar, 100 $\mu \mathrm{m}$. E, The number of oligodendrocytes is reduced in $\mathrm{fyn}^{-/-}$corpus callosum but not in spinal cord. Oligodendrocytes were counted in cervical spinal cord and corpus callosum of control and $\mathrm{fyn}^{-1-}$ mice, which expressed $\beta$-galactosidase from the $M b p$-lacZ transgene. In spinal cord, every oligodendrocyte was counted in 10 nonadjacent $6-\mu \mathrm{m}$-thick crosssections of the cervical enlargement of the spinal cord. The number of oligodendrocytes in corpus callosum was determined by counting oligodendrocytes in five nonadjacent $6-\mu \mathrm{m}$ thick coronal cross-sections. Counts were made in $\mathrm{yyn}^{-1-}$ and control sections at corresponding levels along the rostrocaudal axis of the corpus callosum. The cross-sectional areas of spinal cord and corpus callosum were determined by capturing a light microscope image of the structure with a digital camera and determining its area in pixels, using Adobe Photoshop. A microruler was observed and measured by the same procedure to convert area in pixels to square millimeters. There was a significant reduction in the number of oligodendrocytes per square millimeter in $f y^{-1-}$ mouse corpus callosum $(p<0.005)$ but not in spinal cord $(p>$ $0.5)($ left $)$. Similarly, there was no reduction in the number of $f y^{-1-}$ spinal cord oligodendro-

protein (Stein et al., 1992). We demonstrated that Fyn is unique among Src kinases in that it is necessary for normal forebrain myelination, whereas the closely related molecules Src, Yes, and Lyn are not. We have further demonstrated that the corpus callosum of $f y n^{-1-}$ mice has fewer oligodendrocytes and drastically reduced numbers of myelin sheaths, whereas spinal cord has a normal number of oligodendrocytes and is normally myelinated. Finally, we show that Fyn kinase activity is required for normal myelination.

\section{The myelin deficit in $\mathrm{fyn}^{-/-}$mice varies by CNS region}

Using several different approaches, analysis of myelin content, analysis of MBP content, and light and electron microscopy, we demonstrated that the $f y n^{-1-}$ forebrain is hypomyelinated. Surprisingly, the myelin in cervical spinal cord appears unaffected. At all ages analyzed, there were no evident abnormalities in myelination of the spinal cord in $f y n^{-/-}$mice, demonstrating that the time of onset and rate of myelination are not affected. Our

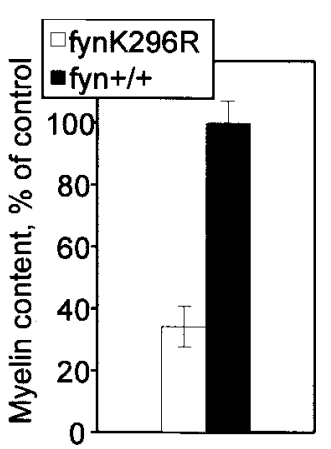

Figure 6. Fyn kinase activity is required for normal myelination. Myelin was isolated and quantified from kinase-deficient FynK296R mutant and age-matched B6 control mouse forebrain at $28 \mathrm{~d}$ of age. Myelin content, calculated as milligrams of myelin (dry weight) per gram of brain (wet weight), is shown as percent of control. Each bar represents the mean \pm SEM value from six animals. 
observation of normal myelination in spinal cord is in disagreement with a recent report that myelin sheath thickness is dramatically decreased in the spinal cord of mice expressing a different Fyn allele (Umemori et al., 1999). Because different tracts within the spinal cord are myelinated at different times during development (Schwab and Schnell, 1989) and because the timing of myelination is highly dependent on position along the length of the spinal cord (Schwab and Schnell, 1989), we made all of our measurements in the ventral funiculus within the cervical enlargement. Because the anatomical location of the measurements was not specified in the previous study (Umemori et al., 1999), it is unclear whether the differences in myelination were attributable to the effect of the fyn mutation or simply to normal developmental differences. Alternatively, it is possible that the abnormal myelin morphology in the spinal cord from the fyn mutant mouse described by Umemori et al. (1999) represents a more severe phenotype resulting from the expression, in those mice, of a truncated Fyn protein that may have dominant-negative or toxic activity, which would not be present in our $f y n^{-1-}$ mice that lack any expression of Fyn protein (Stein et al., 1992).

Despite normal myelin content within the spinal cord of $f y n^{-/-}$ mice, we found a large deficit in forebrain. Analysis of $f y n^{-1-}$ corpus callosum revealed a $29 \%$ reduction in the number of oligodendrocytes and a more extensive, $77 \%$, reduction in the number of myelinated fibers. This discrepancy suggests that the $f y n^{-1-}$ oligodendrocytes in the corpus callosum are deficient in their ability to myelinate the appropriate number of axons. This possibility is consistent with the observation that Fyn is important for the development of the extensive network of processes characteristic of mature oligodendrocytes (Osterhout et al., 1999; Sperber and McMorris, 2001). Thus, a deficit in process outgrowth could result in a decrease in the number of axons that each oligodendrocyte could successfully myelinate. Three oligodendroglial cell-surface molecules have been identified that may contribute to this oligodendrocyte-autonomous phenotype: MAG (Umemori et al., 1994), NCAM120, and F3 (Kramer et al., 1999). In addition, Fyn is required for normal insulin-like growth factor I signaling in oligodendrocytes (Sperber and McMorris, 2001). It is possible that the absence of Fyn results in deficient signaling through some or all of these adhesion and signaling molecules, thus disrupting normal oligodendrocyte function.

Recently, Biffiger et al. (2000) reported that the optic nerve of $\mathrm{fyn}^{-/-}$mice is hypomyelinated, consistent with our observation of a significant decrease in optic nerve cross-sectional area and of forebrain hypomyelination, and also reported that myelin sheaths of $f y n^{-/-}$mice are indistinguishable from control by light and electron microscopy, in agreement with our findings. No apparent reduction in oligodendrocyte number was observed in optic nerve, similar to our observation in spinal cord but very different from our observation in corpus callosum. Additionally, in doublemutant mice $\left(\mathrm{fyn}^{-/-} / \mathrm{mag}^{-/-}\right)$, Biffiger et al. (2000) observed regional differences in phenotype; myelination was unaffected in fasciculus gracilis and cuneatus or ventral funiculus of spinal cord but was reduced in corticospinal tract and optic nerve.

It is not clear, however, why the absence of Fyn from oligodendrocytes would give rise to a dramatic myelin deficiency within some regions of CNS (corpus callosum) and not others (spinal cord). Although the initiation of myelination occurs in the spinal cord at a much earlier time than in the corpus callosum, we found no myelin abnormalities, even at early stages of myelination in spinal cord; therefore, the regional variation cannot be attributed to intrinsic differences in the timing of myelination. Interestingly, mice deficient in platelet-derived growth factor A (PDGF-A) exhibit a different pattern of regional differences: $P D G F-A$ null mice are more severely hypomyelinated in optic nerve and spinal cord than in corpus callosum and cerebral cortex, the reverse of what we observed in our fyn null mice (Fruttiger et al., 1999).

One possible explanation for the regional differences we observed is that oligodendrocytes in forebrain require Fyn for normal myelination, whereas those in spinal cord do not. In classic morphological studies, Del Rio-Hortega (1928) proposed that there are distinct subpopulations of oligodendroglia, and there are additional findings consistent with this view. Forebrain and spinal cord oligodendrocytes have distinct morphologies in neuron-free cultures, those in forebrain having a greater number of processes (Bjartmar, 1998). Evidence has been presented for two independent populations of oligodendrocyte precursors, one characterized by expression of PDGF $\alpha$-receptors and the other by expression of mRNA for the myelin protein DM20 (Spassky et al., 1998, 2000) (but see Fruttiger et al., 1999). If there are separate lineages of oligodendrocytes, they may differ in the growth factors and signaling pathways that regulate their development and function.

However, most evidence indicates that local neuronal factors dictate which and how many axons are myelinated, as well as the amount of myelin they receive. When optic nerve oligodendrocytes, which ordinarily myelinate numerous small axons, are transplanted into spinal cord, they myelinate only a few large axons (Fanarraga et al., 1998). Additionally, the formation of compact myelin does not occur in the absence of axons (Lubetzki et al., 1993). Thus, we must consider what role neurons might be playing in the $f y n^{-1-}$ mouse myelin phenotype. Because neurons also express relatively high levels of Fyn and because there is an intimate reciprocal regulation between oligodendrocytes and neurons, we cannot rule out the possibility that the lack of Fyn in neurons contributes to the hypomyelinating phenotype, and the regional differences in myelination arise from neuronal influences. Nonetheless, because Fyn is active in oligodendrocytes (Osterhout et al., 1999) and is important for the normal morphological development of oligodendrocytes in the absence of neurons (Osterhout et al., 1999; Sperber and McMorris, 2001), we believe that the myelin deficit of $f y n^{-1-}$ mice results, at least in part, from the absence of Fyn in oligodendrocytes.

\section{The role of Fyn in myelination is unique among the Src family of kinases}

Of the nine known Src family kinase members, five family members, Src, Fyn, Yes, Lyn, and Lck, are expressed in brain (Thomas and Brugge, 1997); of these, Fyn (Umemori et al., 1994; Osterhout et al., 1999) and Lyn (Osterhout et al., 1999) have been reported to be expressed within oligodendrocytes. A recent study performed in rat was unable to detect Src or Yes in oligodendrocytes (Osterhout et al., 1999), but we detected expression of Src in purified mouse oligodendroglial cells. The apparent discrepancy in expression of Src is likely attributable to differences in the methods rather than to species differences. Taking these data together, at least three members of the Src family, Fyn, Lyn, and Src, are expressed in oligodendrocytes. However, we found that, in sharp contrast to the striking myelin deficit when Fyn is absent, there is no detectable deficit in CNS myelination in the absence of Lyn, Src, or Yes.

\section{Fyn kinase activity is required for normal myelination}

Fyn and the other Src family members possess protein tyrosine kinase activity that plays a prominent role in their function as 
signaling molecules. In comparison, many important signal transduction molecules are devoid of kinase or other known enzymatic activity but instead act as adapter proteins; by virtue of $\mathrm{SH}$, $\mathrm{SH}$, or other binding motifs, they assemble other proteins into specific functional signaling complexes. Src family kinases, and indeed many other signaling molecules with catalytic activity, also have binding motifs, such as $\mathrm{SH} 2$ and $\mathrm{SH} 3$ domains, and there is accumulating evidence that they can perform signaling functions independent of their enzymatic activity (Xu and Littman, 1993; Kaplan et al., 1995; Schwartzberg et al., 1997; Katsuta et al., 1998). In addition to its kinase domain, Fyn has an SH2, an SH3, and a unique domain, all of which are believed to be important for normal Fyn activity. In the case of CNS myelination, however, we have shown that full-length, catalytically inactive Fyn is not sufficient to rescue the $f y n^{-1-}$ mouse myelin deficit, indicating that the function of Fyn in myelination requires its kinase activity.

\section{REFERENCES}

Bare DJ, Lauder JM, Wilkie MB, Maness PF (1993) p59 fyn in rat brain is localized in developing axonal tracts and subpopulations of adult neurons and glia. Oncogene 8:1429-1436.

Barres BA, Hart IK, Coles HS, Burne JF, Voyvodic JT, Richardson WD, Raff MC (1992) Cell death and control of cell survival in the oligodendrocyte lineage. Cell 70:31-46.

Biffiger K, Bartsch S, Montag D, Aguzzi A, Schachner M, Bartsch U (2000) Severe hypomyelination of the murine CNS in the absence of myelin-associated glycoprotein and Fyn tyrosine kinase. J Neurosci 20:7430-7437.

Bjartmar C (1998) Morphological heterogeneity of cultured spinal and cerebral rat oligodendrocytes. Neurosci Lett 247:91-94.

Brown MT, Cooper JA (1996) Regulation, substrates and functions of src. Biochim Biophys Acta 1287:121-149.

Chan VW, Meng F, Soriano P, DeFranco AL, Lowell CA (1997) Characterization of the B lymphocyte populations in Lyn-deficient mice and the role of Lyn in signal initiation and down-regulation. Immunity 7:69-81.

Del Rio-Hortega P (1928) Tercera aportacion al conocimiento morfologica e interpretacion functional de la oligodendroglia. Mem Real Soc Exapn Hist Nat 14:5-122.

Eisenbarth GS, Walsh FS, Nirenberg M (1979) Monoclonal antibody to a plasma membrane antigen of neurons. Proc Natl Acad Sci USA 76:4913-4917.

Fanarraga ML, Griffiths IR, Zhao M, Duncan ID (1998) Oligodendrocytes are not inherently programmed to myelinate a specific size of axon. J Comp Neurol 399:94-100.

Fruttiger, M, Karlsson, L, Hall AC, Abramsson, A, Calver AR, Bostrom, H, Willetts, K, Bertold CH, Heath JK, Betsholtz, C, Richardson WD (1999) Defective oligodendrocyte development and severe hypomyelination in PDGF-A knockout mice. Development 126:457-467.

Grant SG, O'Dell TJ, Karl KA, Stein PL, Soriano P, Kandel ER (1992) Impaired long-term potentiation, spatial learning, and hippocampal development in fyn mutant mice. Science 258:1903-1910.

Kaplan KB, Swedlow JR, Morgan DO, Varmus HE (1995) c-Src enhances the spreading of $s r c-/-$ fibroblasts on fibronectin by a kinaseindependent mechanism. Genes Dev 9:1505-1517.

Katsuta H, Tsuji S, Niho Y, Kurosaki T, Kitamura D (1998) Lynmediated down-regulation of B cell antigen receptor signaling: inhibition of protein kinase $\mathrm{C}$ activation by Lyn in a kinase-independent fashion. J Immunol 160:1547-1551.

Kramer EM, Klein C, Koch T, Boytinck M, Trotter J (1999) Compartmentation of Fyn kinase with glycosylphosphatidylinositol-anchored molecules in oligodendrocytes facilitates kinase activation during myelination. J Biol Chem 274:29042-29049.
Lees MB, Brown D (1984) Proteins in myelin. In: Myelin (Morell P, ed), pp 197-224. New York: Plenum.

Lubetzki C, Demerens C, Anglade P, Villarroya H, Frankfurter A, Lee VM, Zalc B (1993) Even in culture, oligodendrocytes myelinate solely axons. Proc Natl Acad Sci USA 90:6820-6824.

McCarthy KD, de Vellis J (1980) Preparation of separate astroglial and oligodendroglial cell cultures from rat cerebral tissue. J Cell Biol 85:890-902.

McMorris FA, Miller SL, Pleasure D, Abramsky O (1981) Expression of biochemical properties of oligodendrocytes in oligodendrocyte $\times$ glioma cell hybrids proliferating in vitro. Exp Cell Res 133:395-404.

Miyakawa T, Yagi T, Watanabe S, Niki H (1994) Increased fearfulness of Fyn tyrosine kinase deficient mice. Brain Res Mol Brain Res 27:179-182.

Miyakawa T, Yagi T, Kitazawa H, Yasuda M, Kawai N, Tsuboi K, Niki H (1997) Fyn-kinase as a determinant of ethanol sensitivity: relation to NMDA-receptor function. Science 278:698-701.

Norton WT, Poduslo SE (1973) Myelination in rat brain: method of myelin isolation. J Neurochem 21:749-757.

Osterhout DJ, Wolven A, Wolf RM, Resh MD, Chao MV (1999) Morphological differentiation of oligodendrocytes requires activation of Fyn tyrosine kinase. J Cell Biol 145:1209-1218.

Raible DW, McMorris FA (1990) Induction of oligodendrocyte differentiation by activators of adenylate cyclase. J Neurosci Res 27:43-46.

Schwab ME, Schnell L (1989) Region-specific appearance of myelin constituents in the developing rat spinal cord. J Neurocytol 18:161-169.

Schwartzberg PL, Xing L, Hoffmann O, Lowell CA, Garrett L, Boyce BF, Varmus HE (1997) Rescue of osteoclast function by transgenic expression of kinase-deficient Src in $s r c-/-$ mutant mice. Genes Dev 11:2835-2844.

Sommer I, Schachner M (1981) Monoclonal antibodies (O1 to O4) to oligodendrocyte cell surfaces: an immunocytological study in the central nervous system. Dev Biol 83:311-327.

Soriano P, Montgomery C, Geske R, Bradley A (1991) Targeted disruption of the $c$-src proto-oncogene leads to osteopetrosis in mice. Cell 64:693-702.

Spassky N, Goujet-Zalc C, Parmantier E, Olivier C, Martinez S, Ivanova A, Ikenaka K, Macklin W, Cerruti I, Zalc B, Thomas JL (1998) Multiple restricted origin of oligodendrocytes. J Neurosci 18:8331-8343.

Spassky N, Olivier C, Perez-Villegas E, Goujet-Zalc C, Martinez S, Thomas JL, Zalc B (2000) Single or multiple oligodendroglial lineages: a controversy. Glia 29:143-148.

Sperber BR, McMorris FA (2001) Fyn tyrosine kinase regulates oligodendroglial cell development but is not required for morphological differentiation of oligodendrocytes. J Neurosci Res 63:303-312.

Stein PL, Lee HM, Rich S, Soriano P (1992) pp59 $9^{\text {fyn }}$ mutant mice display differential signaling in thymocytes and peripheral $\mathrm{T}$ cells. Cell 70:741-750.

Stein PL, Vogel H, Soriano P (1994) Combined deficiencies of Src, Fyn, and Yes tyrosine kinases in mutant mice. Genes Dev 8:1999-2007.

Thomas SM, Brugge JS (1997) Cellular functions regulated by Src family kinases. Annu Rev Cell Dev Biol 13:513-609.

Thomas SM, Soriano P, Imamoto A (1995) Specific and redundant roles of Src and Fyn in organizing the cytoskeleton. Nature 376:267-271.

Umemori H, Sato S, Yagi T, Aizawa S, Yamamoto T (1994) Initial events of myelination involve Fyn tyrosine kinase signalling. Nature $367: 572-576$.

Umemori H, Kadowaki Y, Hirosawa K, Yoshida Y, Hironaka K, Okano H, Yamamoto T (1999) Stimulation of myelin basic protein gene transcription by Fyn tyrosine kinase for myelination. J Neurosci 19:1393-1397.

Vemuri GS, McMorris FA (1996) Oligodendrocytes and their precursors require phosphatidylinositol 3-kinase signaling for survival. Development 122:2529-2537.

$\mathrm{Xu}$ H, Littman DR (1993) A kinase-independent function of Lck in potentiating antigen-specific $\mathrm{T}$ cell activation. Cell 74:633-643.

Yagi T, Shigetani Y, Okado N, Tokunaga T, Ikawa Y, Aizawa S (1993) Regional localization of Fyn in adult brain; studies with mice in which fyn gene was replaced by lacZ. Oncogene 8:3343-3351. 